Ciencia y Salud, Vol. V, No. 2, mayo-agosto, 2021 • ISSN (impreso): 2613-8816 • ISSN (en línea): 2613-8824

DOI: https://doi.org/10.22206/cysa.2021.v5i2.pp19-26

\title{
FACTORES PATOLÓGICOS ASOCIADOS A DEMENCIA SENIL EN ADULTOS MAYORES DE UN CENTRO MÉDICO
}

\section{Pathological factors associated with senile dementia in older adults at a Medical Center}

\section{Katia Liseth Vásquez Edquén ${ }^{\mathrm{a}}$ y José Ander Asenjo-Alarcón ${ }^{\mathrm{b}}$}

Recibido: 6 de junio, 2020 • Aprobado: 22 de agosto, 2020

Cómo citar: Vásquez Edquén KL, Asenjo-Alarcón JA. Factores patológicos asociados a demencia senil en adultos mayores de un centro médico. cysa [Internet]. 8 de junio de 2021 [citado 16 de junio de 2021];5(2):19-6. Disponible en: https://revistas.intec.edu.do/ index.php/cisa/article/view/2209

\section{Resumen}

Introducción: la demencia senil es una patología degenerativa, en la actualidad su frecuencia está incrementándose en adultos mayores, debido, esencialmente, a comorbilidades o complicaciones inherentes a su condición fisiológica; las investigaciones apuntan a que su ocurrencia se asocia a hipertensión arterial y diabetes mellitus tipo 2 .

Objetivo: la investigación tuvo como objetivo comparar la demencia senil presuntiva en adultos mayores con y sin factores patológicos del Centro Médico EsSalud de Chota, Cajamarca.

Metodología: el estudio fue relacional, transversal, realizado en 178 adultos mayores atendidos en el Centro Médico EsSalud, se utilizó una ficha de factores patológicos y el Test Mini Mental.

Resultados: como factores patológicos, el 39,9 \% de adultos mayores presentaron hipertensión arterial de leve a severa y el $21,9 \%$ presentaron diabetes mellitus tipo 2 . El 84,3\% de adultos mayores presentaron demencia senil presuntiva de leve a severa.

\footnotetext{
a Universidad Nacional Autónoma de Chota, Cajamarca, Perú.

Correo-e: katicita1997@hotmail.com
}

\begin{abstract}
Introduction: Senile dementia is a degenerative pathology, currently its frequency is increasing in older adults, essentially due to comorbidities or complications inherent to their physiological condition; Research indicates that its occurrence is associated with hypertension and type 2 diabetes mellitus.

Objective: The objective of the research was to compare presumptive senile dementia in older adults with and without pathological factors from the EsSalud Medical Center in Chota, Cajamarca.

Methodology: The study was relational, cross-sectional, carried out in 178 older adults treated at the EsSalud Medical Center, a file of pathological factors and the Mini Mental Test were used.

Results: As pathological factors, 39.9\% of older adults had mild to severe hypertension and $21.9 \%$ had type 2 diabetes mellitus. $84.3 \%$ of older adults had mild to severe presumptive senile dementia.

\footnotetext{
b Doctor en Ciencias de la Salud. Docente de la Universidad Nacional Autónoma de Chota, Cajamarca, Perú. ORCID: 0000-0002-1059-4258,
} Correo-e: jasenjo@unach.edu.pe
\end{abstract}


Conclusión: se concluye que existen diferencias estadísticas significativas entre la demencia senil presuntiva en adultos mayores con y sin factores patológicos (hipertensión arterial $[\mathrm{p}=0,000]$ y diabetes mellitus tipo $2[\mathrm{p}=0,000])$.

Palabras clave: hipertensión; diabetes mellitus tipo 2; Enfermedad de Alzheimer; disfunción cognitiva; adulto mayor. (DeCS BIREME).

\section{Introducción}

La demencia senil es un síndrome que ocasiona un deterioro cognitivo progresivo y constante de la memoria, lenguaje, intelecto, orientación, cálculo o percepción espacial, comportamiento y capacidad para realizar las actividades de la vida diaria, provocando pérdida de la autonomía en los adultos mayores; está asociada principalmente a patologías crónicas como la hipertensión arterial (HTA) y la diabetes mellitus tipo 2 (DM2). ${ }^{1,2}$.

Organismos internacionales han propuesto el plan de acción mundial sobre la respuesta de salud pública hacia la demencia, con la finalidad de abordar su evaluación, la concientización, reducción de riesgos, diagnóstico, tratamiento, sistemas de información e investigación. Asimismo, la Organización Panamericana de la Salud (OPS) reconoce a la HTA y DM2 como factores asociados a la demencia senil y brinda recomendaciones a partir de evidencia científica para el manejo adecuado de los mismos. ${ }^{2,3}$

Las cifras mundiales de demencia senil son exorbitantes, su afectación alcanza 50 millones de personas, en promedio el $60 \%$ de ellas pertenecen a países de ingresos bajos y medios. Por año las cifras alcanzan 10 millones de nuevos casos y se calcula que entre $5 \%$ y $8 \%$ de adultos mayores de 60 ańos padecen de demencia senil en algún momento de sus vidas; se estima que el número total de personas con demencia senil alcanzará 82 millones en el año 2030 y 152 millones en el año $2050^{2}$.
Conclusion: It is concluded that there are statistically significant differences between presumptive senile dementia in older adults with and without pathological factors (arterial hypertension $[\mathrm{p}=0.000]$ and type 2 diabetes mellitus $[\mathrm{p}=0.000])$.

Keywords: Hypertension; diabetes mellitus, type 2; Alzheimer Disease; cognitive dysfunction; aged. (MeSH NLM).

Los factores patológicos ligados a la demencia senil prioritariamente son la HTA y la DM2; en este sentido, la DM2 constituye un factor de riesgo para la progresión de la demencia vascular y el deterioro cognitivo leve en adultos mayores, provocándoles obnubilación del pensamiento y de la memoria. Investigaciones apuntan que la DM2 puede incrementar el riesgo de que el deterioro cognitivo leve avance hasta la demencia. Deterioro que puede anteceder a la enfermedad de Alzheimer y otros tipos de demencia .

Por su parte, la HTA también se asocia a padecimientos demenciales; por lo que, si un paciente mantiene una presión arterial elevada por mucho tiempo puede acarrearle micro lesiones cerebrales, capaces de provocar un daño permanente y el desarrollo de algún grado de demencia 5 .

En el contexto regional y local, la atención que se brinda al adulto mayor es escasa y se le resta importancia a los cambios fisiológicos progresivos que experimentan con el paso de los años. Los cambios fisiológicos que afectan el sistema nervioso de los adultos mayores, pueden acentuarse debido a comorbilidades como la HTA y DM2, las que requieren ser identificadas y controladas favorablemente en su avance, para atenuar el impacto en el desarrollo de la demencia senil. La existencia de escasos estudios a nivel regional entre factores patológicos y demencia senil en adultos mayores, resultó muy relevante para el desarrollo de la investigación. 
El estudio tuvo como objetivo comparar la demencia senil presuntiva en adultos mayores con y sin factores patológicos del Centro Médico EsSalud de Chota, Cajamarca.

\section{Material y métodos}

Estudio relacional, transversal, realizado durante los meses de agosto a octubre del año 2017. La población estudiada fue de 178 adultos mayores de ambos sexos, atendidos en el Centro Médico EsSalud de Chota, con edades comprendidas entre 60 y 85 años de edad.

Las técnicas de recolección de datos fueron el análisis documental para los factores patológicos (HTA y DM2) y la encuesta para la demencia senil presuntiva. Para determinar los factores patológicos (HTA y DM2) se utilizó una ficha de recolección de datos adaptada del Ministerio de Salud ${ }^{6}$ y la Asociación Americana de Diabetes ${ }^{7}$, con valores: Presión arterial normal (< 120/80 mm Hg), HTA leve $(140 / 90 ; 159 / 99 \mathrm{~mm} \mathrm{Hg})$, HTA moderada $(160 / 100 ; 179 / 109$ mm Hg), HTA severa ( $\geq 180 / 110$ $\mathrm{mm} \mathrm{Hg}$ ) y el diagnóstico médico de DM2. La demencia senil presuntiva fue evaluada a través del Test Mini Mental de Folstein, creado en Estados Unidos y adaptado a Perú por Custodio y Lira ${ }^{8}$, consta de 11 ítems y evalúa 5 dimensiones: Orientación temporal-espacial (de 0 a 10 puntos), fijación recuerdo (de 0 a 3 puntos), atención cálculo (de 0 a 5 puntos), recuerdo diferido (de 0 a 3 puntos) y lenguaje (de 0 a 9 puntos). El puntaje global se clasifica en: de 0 a 18 puntos demencia severa, de 19 a 22 puntos demencia leve a moderada y de 23 a 30 puntos sin deterioro.

Para adaptar el test al contexto local, se realizó una prueba piloto con 20 adultos mayores atendidos en la Sanidad de la Policía Nacional del Perú de Chota, los que presentaban las mismas características de las unidades de estudio. El análisis de fiabilidad mediante la prueba Alfa de Crombach fue de 0,84 , adecuado para ser aplicado en la población de estudio.

\section{Procedimiento de recolección de datos}

Para la recolección de datos se solicitó permiso al director del Centro Médico EsSalud de Chota y al enfermero responsable del Programa del Adulto Mayor, quienes firmaron la autorización y proporcionaron la información necesaria. La información de los factores patológicos (HTA y DM2) se recolectó de la historia clínica a través de una ficha. Posteriormente, se realizaron las visitas a los adultos mayores en sus domicilios; se les instó a participar en la investigación mediante una carta de invitación y exposición de los objetivos, quienes asintieron firmaron el consentimiento informado, para luego aplicarles el Test Mini Mental.

\section{Análisis estadístico}

La información recolectada se ingresó en una matriz de datos creada en el software estadístico SPSS v. 24 para Windows. El análisis estadístico univariado se realizó mediante frecuencias absolutas y relativas. El análisis estadístico bivariado se realizó mediante la prueba estadística U de Mann - Whitney, siendo estadísticamente significativo un p-valor $<0,05$.

\section{Aspectos éticos}

Los principios éticos fueron puestos en práctica durante todo el proceso investigativo. Los adultos mayores de manera voluntaria y bien informada aceptaron participar en el estudio y firmaron el consentimiento informado de manera directa o a través de un familiar.

\section{Resultados y discusión}

El estudio se realizó con 178 adultos mayores de ambos sexos, con edades comprendidas entre $60 \mathrm{y}$ 85 años, atendidos en el Centro Médico EsSalud de Chota. Como factores patológicos, el 39,9 \% de 
adultos mayores presentaron HTA de leve a severa y el 21,9\% presentaron DM2 (tabla 1). El 84,3\% de adultos mayores presentaron demencia senil presuntiva de leve a severa (tabla 2).
Existen diferencias estadísticas significativas entre la demencia senil presuntiva en adultos mayores con y sin factores patológicos (HTA $[\mathrm{p}=0,000]$ y DM2 $[\mathrm{p}=0,000])$ (tabla 3$)$.

Tabla 1. Factores patológicos en adultos mayores del Centro Médico EsSalud de Chota

\begin{tabular}{|c|c|c|c|c|c|c|}
\hline \multirow{2}{*}{$\begin{array}{c}\text { Factores patoló- } \\
\text { gicos }\end{array}$} & \multicolumn{2}{|c|}{ F } & \multicolumn{2}{|c|}{ Sexo } & \multicolumn{2}{c|}{ Total } \\
\cline { 2 - 7 } & $\mathbf{n}(\mathbf{9 6})$ & $\mathbf{9}$ & $\mathbf{n}(\mathbf{8 2})$ & $\%$ & $\mathbf{n}(\mathbf{1 7 8 )}$ & $\%$ \\
\hline $\begin{array}{c}\text { Hipertensión arte- } \\
\text { rial }\end{array}$ & & & & & & \\
\hline Severa & 8 & 8,3 & 5 & 6,2 & 13 & 7,3 \\
\hline Moderada & 7 & 7,3 & 7 & 8,5 & 14 & 7,9 \\
\hline Leve & 22 & 22,9 & 22 & 26,8 & 44 & 24,7 \\
\hline $\begin{array}{c}\text { Presión arterial } \\
\text { normal }\end{array}$ & 59 & 61,5 & 48 & 58,5 & 107 & 60,1 \\
\hline $\begin{array}{c}\text { Diabetes mellitus } \\
\text { tipo 2 }\end{array}$ & & & & & & \\
\hline Presenta & 21 & 21,9 & 18 & 21,9 & 39 & 21,9 \\
\hline No presenta & 75 & 78,1 & 64 & 78,0 & 139 & 78,1 \\
\hline
\end{tabular}

Fuente: elaboración propia.

Tabla 2. Demencia senil presuntiva en adultos mayores del Centro Médico EsSalud de Chota

\begin{tabular}{|l|c|c|c|c|c|c|}
\hline \multirow{2}{*}{ Demencia senil presuntiva } & \multicolumn{4}{|c|}{ Sexo } & \multicolumn{3}{c|}{ Total } \\
\cline { 2 - 8 } & F & \multicolumn{2}{c|}{ M } & \multicolumn{2}{c|}{} \\
\cline { 2 - 8 } & n (96) & $\%$ & n (82) & $\%$ & n (178) & $\%$ \\
\hline Severa & 53 & 55,2 & 49 & 59,8 & 102 & 57,3 \\
\hline Leve a moderada & 32 & 33,3 & 16 & 19,5 & 48 & 27,0 \\
\hline Sin deterioro & 11 & 11,5 & 17 & 20,7 & 28 & 15,7 \\
\hline
\end{tabular}

Fuente: elaboración propia.

22 | Ciencia y Salud 2021; 5(2, mayo-agosto): 19-26• Artículo original 
Tabla 3. Factores patológicos y demencia senil presuntiva en adultos mayores del Centro Médico EsSalud de Chota

\begin{tabular}{|c|c|c|c|c|c|c|c|}
\hline \multirow{3}{*}{ Factores patológicos } & \multicolumn{6}{|c|}{ Demencia senil presuntiva } & \multirow{3}{*}{ p-valor } \\
\hline & \multicolumn{2}{|c|}{ Severa } & \multicolumn{2}{|c|}{ Leve a moderada } & \multicolumn{2}{|c|}{ Sin deterioro } & \\
\hline & n (102) & $\%$ & n (48) & $\%$ & n (28) & $\%$ & \\
\hline \multicolumn{8}{|l|}{ Hipertensión arterial } \\
\hline Presenta & 45 & 63,4 & 16 & 22,5 & 10 & 14,1 & \multirow{2}{*}{$0,000^{*}$} \\
\hline No presenta & 57 & 53,3 & 32 & 29,9 & 18 & 16,8 & \\
\hline \multicolumn{8}{|l|}{ Diabetes mellitus tipo 2} \\
\hline Presenta & 22 & 56,4 & 13 & 33,3 & 04 & 10,3 & \multirow{2}{*}{$0,000^{*}$} \\
\hline No presenta & 80 & 53,3 & 35 & 29,9 & 24 & 16,8 & \\
\hline
\end{tabular}

*U de Mann - Whitney $(\mathrm{p}<0,05)$.

Fuente: elaboración propia.

La HTA se presentó en más de la tercera parte de adultos mayores, debido a que su ocurrencia es más frecuente en edades avanzadas y por ser una enfermedad silenciosa, muchas veces pasa desapercibida por el paciente y los familiares. Resultados un tanto parecidos reporta Montes ${ }^{9}$ con el $8,69 \%$ de adultos mayores de 60 a 70 ańos con HTA, a diferencia de nuestro estudio que se abarcó hasta los 85 años; así mismo, Peñaherrera ${ }^{10}$ encontró que el 14,45 \% de adultos mayores presentaban HTA, con mayor frecuencia a los 80 años. Chandía y Luengo ${ }^{11}$ también reportan resultados considerables en la frecuencia de HTA en los adultos mayores, con el $50 \%$, con mayor concentración entre las edades de 70 a 79 años.

La HTA incrementa su frecuencia conforme avanza la edad de las personas, siendo los adultos mayores el grupo más proclive de padecerla, constituyéndose en un importante factor de riesgo cardiovascular y neurológico. La HTA sistólica aislada es la forma dominante, debido al endurecimiento arterial creciente y a la acumulación de ateromas en las paredes de los vasos arteriales por la hipertrofia y esclerosis de su capa muscular ${ }^{12}$.

Los resultados del aumento progresivo de la presión arterial en los adultos mayores, generalmente están relacionados con el consumo de alimentos ricos en sodio y grasas saturadas, pobre ingesta de frutas y verduras, consumo frecuente de bebidas alcohólicas, sedentarismo, inactividad física y estrés. Las condiciones de vida y trabajo juegan un rol muy preponderante en la práctica de estilos de vida no saludables. El hábito tabáquico y la HTA están ligadas en el incremento de la probabilidad de ocurrencia de enfermedades cardiovasculares ${ }^{13}$.

La DM2 se presentó en más de la quinta parte de adultos mayores, es tan frecuente como la HTA y generalmente se manifiestan juntas en un mismo paciente, debido a que sus mecanismos fisiopatológicos son 
muy comunes. Resultados similares reportan Beobide-Telleria et al. ${ }^{14}$, con el $21,7 \%$ de adultos mayores con DM2, debido a que esta enfermedad está propagada en diferentes contextos sociodemográficos. Así mismo Ruiz-García et al. ${ }^{15}$, encontraron que la prevalencia de DM2 en los adultos mayores de 70 o más años $(30,3 \%)$ es el doble que en los adultos de $40 \mathrm{y}$ 69 años (15,3\%), magnificando de esta manera la gravedad de la DM2, información que debe ser tenida en cuenta en las políticas de salud de la población.

La pirámide poblacional está mostrando un ascenso en el grupo de los adultos mayores, junto a ello, también ya existe un incremento progresivo de las enfermedades crónicas como la HTA y la DM2; esta última asociada con factores físicos, cognoscitivos y sociofamiliares, producto de comorbilidades y carga de enfermedad acumulada a lo largo de los años, más aún si el adulto mayor en etapas anteriores ha llevado estilos de vida inadecuados. En este sentido, se requiere el compromiso serio del paciente y familiares para un abordaje integral que coadyuve al control de la enfermedad y retraso de las complicaciones consecuentes. ${ }^{16}$

La DM2 es una de las patologías más preponderantes en los adultos mayores, y repercute desfavorablemente en las condiciones de vida del paciente y los familiares; sumado a ella pueden presentarse: el síndrome de fragilidad y el deterioro cognitivo, que agravan aún más la situación mermando su calidad de vida y originando costes directos e indirectos para la población y el sistema de salud. ${ }^{17}$

Respecto a la demencia senil presuntiva, ocho de cada diez adultos mayores la presentan en diferente grado, siendo estadísticamente significativa su presencia en adultos mayores con HTA y DM2. Ramos et al. ${ }^{18}$ reportan datos opuestos respecto al deterioro cognitivo, con $10 \%$ en el nivel moderado. En nuestro estudio representó el $27 \%$. También aducen que los adultos mayores que tuvieron de seis a diez años de diagnóstico de DM2 reportaron $5 \%$ de deterioro cognitivo leve y aquellos con más de diez años de diagnóstico representaron 5,8\%, encontró que la HTA sistémica fue la comorbilidad mayormente asociada a DM2 para desarrollar deterioro cognitivo grave (4\%). Así mismo, Sotolongo y Alvarez ${ }^{19}$ encontraron que la demencia estuvo presente en el $12,5 \%$ de adultos mayores con DM2.

Concerniente a la relación entre DM2 y demencia senil, Chandía y Luengo ${ }^{11}$ encontraron resultados similares, con una relación estadística significativa entre DM2 y deterioro cognitivo $(\mathrm{p}=0,024)$. Weyman (20), también reporta una relación estadística significativa entre DM2 y deterioro cognitivo. Estos resultados permiten aseverar, que la demencia senil es una comorbilidad frecuente en los adultos mayores con HTA y DM2, indistintamente del contexto sociodemográfico en el que se encuentren.

El deterioro cognitivo afecta esencialmente a la memoria, en tanto que la demencia senil tiene alcance de afectación a las funciones cerebrales, impidiendo el normal desarrollo y desenvolvimiento de los adultos mayores en su cotidianeidad, el deterioro cognitivo es parte de la demencia senil. La demencia senil es el resultado del daño o pérdida de células nerviosas y sus conexiones cerebrales, acelerado por factores como la HTA y DM2. Según el área del cerebro que se encuentre afectada, las manifestaciones clínicas y la gravedad serán diferentes, dependiendo de las características de los adultos mayores ${ }^{21}$.

Como limitación del estudio, la demencia senil debe ser diagnosticada por un especialista; no obstante, lo que se pretende con los resultados de la investigación es presentar un diagnóstico presuntivo de demencia senil asociada a HTA y DM2 en adultos mayores del Centro Médico EsSalud de Chota.

\section{Conclusión}

En conclusión, los factores patológicos de los adultos mayores estudiados fueron la HTA y la DM2. Ocho de cada diez adultos mayores presen- 
taron algún grado de demencia senil, de leve a severa. Finalmente, existen diferencias estadísticas significativas entre la demencia senil presuntiva en adultos mayores con factores patológicos respecto a los que no lo presentan; por lo que se deben establecer las medidas adecuadas de intervención por parte de los actores sociales comprometidos, de manera efectiva y oportuna, para mejorar la calidad de vida de los afectados y familiares.

\section{Bibliográfia}

1. Villarejo A, Eimil M, Llamas S, Llanero M, López C, Prieto C. Informe de la Fundación del cerebro. Impacto social de las enfermedades de Alzheimer y otras demencias. Neurología; 2017:11. Disponible en: https://www.elsevier. es/es-revista-neurologia-295-avance-resumen-informe-fundacion-del-cerebro-impacto-S021348531730350X

2. Organización Mundial de la Salud. Demencia. Ginebra, Suiza: Organización Mundial de la Salud; 2020. Disponible en: https://www.who.int/ es/news-room/fact-sheets/detail/dementia

3. Organización Panamericana delaSalud. Demencia: una prioridad de salud pública. Washington, DC, Estados Unidos: Organización Panamericana de la Salud; 2013. Disponible en: https:// apps.who.int/iris/bitstream/handle/10665/ 98377/9789275318256_spa.pdf;jsessionid= D480E531084636519E21530303D 3D259? sequence $=1$

4. Mayo Clinic. El vínculo entre la diabetes y la enfermedad de Alzheimer. Rochester, Estados Unidos: Mayo Clinic; 2019. Disponible en: https://www.mayoclinic.org/es-es/diseases-conditions/type-2-diabetes/in-depth/diabetes-and-alzheimers/art-20046987

5. Gutiérrez P. ¿Padeces hipertensión? cuidado, podrías sufrir demencia. México: Excelsior; 2019.
Disponible en: https://www.excelsior.com.mx/ trending/padeces-hipertension-cuidado-podrias-sufrir-demencia/1338899\#view-1

6. Ministerio de Salud. Guía de Práctica Clínica para el Diagnóstico, Tratamiento y Control de la Enfermedad Hipertensiva. Lima, Perú: Ministerio de Salud; 2015. Disponible en: https://www. saludarequipa.gob.pe/salud_personas/archivos/ GPC\%202015/RM031-2015-MINSA\%20 Dx\%20tratamiento\%20y\%20Control\%20 de\%20la\%20Enfermedada\%20Hipertensiva.pdf

7. American Diabetes Association. Standards of Medical Care in Diabetes — 2017. Diabetes Care, 2017;40(Supp 1):S1-S135. Disponible en: http:// fmdiabetes.org/wp-content/uploads/2017/12/ ADA__2017.pdf

8. Custodio N,Lira D. Adaptación peruana del Minimental State Examination (MMSE). Anales de la Facultad de Medicina, 2014;75(1):69. Disponible en: http://www.scielo.org.pe/scielo.php?script= sci_arttext\&pid=S1025-55832014000100012

9. Montes JS. (2019). Prevalencia de Hipertensión arterial (HTA) en adultos mayores de 60 a 70 años, atendidos en el Centro de Atención Primaria III Metropolitano EsSalud durante el periodo 2013-2017. Ayacucho 2018 (tesis de licenciatura, Universidad Nacional de San Cristóbal de Huamanga). Disponible en: http://209.45.73.22/ bitstream/handle/UNSCH/3354/TESIS\%20 Far539_Mon.pdf?sequence $=1$ \&isAllowed=y

10. Peñaherrera LA. (2020). Prevalencia y factores de riesgo para hipertensión arterial en adultos mayores en áreas urbanas y rurales del Cantón Ambato (tesis de licenciatura, Universidad Técnica de Ambato). Disponible en: https://repositorio. uta.edu.ec/bitstream/123456789/31113/1/ PE \% c $3 \% 91$ A HERRERA \% 20 L \% c $3 \% 93$ P EZ\% $20 \% 2$ cLIZBET H \% 20 ANDREA\%20\%26.pdf 
11. Chandía VA, Luengo C. Relación entre hipertensión arterial y diabetes mellitus tipo 2 con deterioro cognitivo en adultos mayores. Gerokomos, 2019;30(4):172-75. Disponible en: http:// scielo.isciii.es/pdf/geroko/v30n4/1134-928Xgeroko-30-04-172.pdf

12. Salazar PM, Rotta A, Otiniano F. Hipertensión en el adulto mayor. Revista Médica Herediana, 2016;27(1):60-6. Disponible en: http:/www. scielo.org.pe/pdf/rmh/v27n1/a10v27n1.pdf

13. Osorio-Bedoya EJ, Amariles P. Hipertensión arterial en pacientes de edad avanzada: una revisión estructurada. Revista Colombiana de Cardiología, 2018;25(3):209-21. Disponible en: https://www.sciencedirect.com/science/article/ pii/S012056331830024X

14. Beobide-Telleria I, Martínez-Arrechea S, Ferro-Uriguen A, Alaba-Trueba J. Prevalencia de diabetes mellitus tipo 2 y su tratamiento farmacológico en personas institucionalizadas en centros residenciales. Farmacia Hospitalaria, 2020;44(3):92-5. Disponible en: https://www. sefh.es/fh/195_04breve0111375esp.pdf

15. Ruiz-García A, Arranz-Martínez E, García-Álvarez JC, García-Fernández ME, Palacios-Martínez D, Montero-Costa A, et al. Prevalencia de diabetes mellitus en el ámbito de la atención primaria espańola y su asociación con factores de riesgo cardiovascular y enfermedades cardiovasculares. Estudio SIMETAP-DM. Clínica e Investigación en Arteriosclerosis, 2020;32(1):15-26. Disponible en: https://www.sciencedirect.com/ science/article/pii/S021491681930049X

16. Aliaga-Díaz E, Cuba-Fuentes S, Mar-Meza M. Promoción de la salud y prevención de las enfer- medades para un envejecimiento activo y con calidad de vida. Revista Peruana de Medicina Experimental y Salud Pública, 2016;33(2):31120. Disponible en: https://rpmesp.ins.gob.pe/ index.php/rpmesp/article/view/2143/2243

17. D’Hyver C. Patologías endocrinas más frecuentes en el adulto mayor. Revista dela Facultad de Medicina (México), 2017;60(4):45-57. Disponibleen: http://www.scielo.org.mx/scielo.php?script=sci_ arttext\&pid=S0026-17422017000400045

18. Ramos AC, Vilchis E, Espinoza G, Chaparro ME. Clasificación del deterioro cognitivo en adultos mayores con diabetes mellitus tipo 2 . Atención Familiar, 2020;27(2):61-5. Disponible en: https://www.medigraphic.com/pdfs/ atefam/af-2020/af202b.pdf

19. Sotolongo O, Álvarez S. Comportamiento de la Diabetes Mellitus Tipo 2 en adultos mayores con demencia. Punta Brava, 2018. Panorama. Cuba y Salud, 2020;15(2):14-8. Disponible en: http://revpanorama.sld.cu/index.php/panorama/article/download/1204/pdf_395

20. Weyman Y. (2020). Asociación entre diabetes tipo 2 y deterioro cognitivo en adultos mayores (tesis de maestría, Universidad Juárez del Estado de Durango). Disponible en: http://repositorio. ujed.mx/jspui/bitstream/123456789/84/1/ TESIS\%20Asociaci\%c3\%b3n\%20entre\%20 diabetes $\% 20$ tipo\%202\%20y\%20deterioro\%20 cognitivo.pdf

21. Mayo Clinic. Demencia. Rochester, Estados Unidos: Mayo Clinic; 2020. Disponible en: https://www.mayoclinic.org/es-es/diseasesconditions/dementia/symptoms-causes/ syc-20352013 